\title{
EFFECTIVE LOWER BOUNDS FOR THE NORM OF THE POINCARÉ O-OPERATOR
}

\author{
MARK SHEINGORN
}

\begin{abstract}
Motivated by McMullen's proof of Kra's conjecture that the norm of the Poincare theta operator $\Theta_{q, \Gamma}$ is less than 1 for every $q$ and $\Gamma$ of finite volume, this paper provides explicit lower bounds for this norm. These bounds are sufficient to show that $\left\|\boldsymbol{\Theta}_{q, \Gamma}\right\| \rightarrow 1$ for fixed $\Gamma$ as $q \rightarrow \infty$. Here the difference from 1 is less than $O\left(\frac{(2 \pi e)^{q-2}}{q^{q-2}}\right)$. For $\Gamma(N) \subseteq \Gamma \subseteq \Gamma_{0}(N),\left\|\Theta_{q, \Gamma}\right\| \rightarrow$ 1 for fixed $q$ as $N \rightarrow \infty$. Here the difference from 1 is $O\left(N^{35-q}\right)$. We prove these results by estimating $\frac{\left\|\Theta_{q, \Gamma}\left(f_{p}\right)\right\|_{A_{q}(\Gamma)}}{\left\|f_{p}\right\|_{A_{q}}}$ where the $f_{p}$ are cusp forms of weight $p \leq q-2$. (Thus such functions may in general tend to optimize $\Theta_{q, \Gamma}$.) In the case of the congruence subgroups they are taken to be products of $\Delta$ and Eisenstein series. Effective formulae are presented for all implied constants.
\end{abstract}

This paper has its origin in Curt McMullen's spectacular proof of "Kra's conjecture", which states that the norm of the Poincare theta operator is always less than 1 [M1]. We begin by describing that conjecture.

Let $H$ be the upper half plane and $\Gamma \subset S L(2, R)$ be a Fuchsian group with $H / \Gamma$ having finite volume. That is $\int_{F} \frac{d x d y}{y^{2}}<\infty$ where $F$ is, say a Dirichlet fundamental region for $\Gamma$ in $H . d \mu=\frac{d x d y}{y^{2}}$ is the Poincaré (hyperbolic) metric on the Riemann surface $H / \Gamma$. More generally, define $d \mu_{q}=y^{q-2} d x d y$ so that $d \mu=d \mu_{0}$. Let $q$ be an integer $\geq 2$. Denote $A_{q}=\{f \mid f$ is analytic on $H$, $\left.f(z)=f(z+1), \int_{0}^{\infty} \int_{0}^{1}|f| d \mu_{q}<\infty\right\}$. This is a Banach space with ( $\mathscr{L}^{1}$-like) norm given by $\|f\|_{A_{q}}=\int_{0}^{\infty} \int_{0}^{1}|f| d \mu_{q}$. Let $A_{q}(\Gamma)$ be the set of analytic cusp forms on $\Gamma$.

(From here on we assume $\Gamma$ contains a parabolic element.) This restriction is not essential, but it is used in this paper. We allow it as our applications are to congruence subgroups of $\Gamma(1)$. By conjugation in $S L(2, R)$, we may assume that $\infty$ is a parabolic fixed point with $\left\langle\left(\begin{array}{ll}1 & 1 \\ 0 & 1\end{array}\right)\right\rangle=\Gamma_{\infty}$ its stabilizer in $\Gamma \cdot A_{q}(\Gamma)$ is also a Banach space with norm $\int_{F}|f| d \mu_{q}$.)

Received by the editors April 11, 1989 and, in revised form, February 7, 1990.

1980 Mathematics Subject Classification (1985 Revision). Primary 10D15, 10D12, 30 F35.

Key words and phrases. Poincaré series, congruence subgroup, Eisenstein series.

Research partially supported by a PSC-CUNY grant. 
The Poincare theta operator $\Theta_{q}$ mapping $A_{q}$ onto $A_{q}(\Gamma)$ is given by

$$
\Theta_{q}(f)=\sum_{A \in \Gamma / \Gamma_{\infty}} f(A z) A^{q q} z
$$

It is trivial that $\left\|\Theta_{q}\right\| \leq 1$ and $\mathrm{Kra}$ conjectured that the inequality is strict for any $q$ and $\Gamma$. Although Earle, Kra, Parson [P1, P2], Strebel [St], Wolpert and Parson-Sheingorn [P-S] worked on this, in the end all that was accomplished prior to McMullen was the verification of Kra's conjecture for specific examples.

This paper gives effective (and explicit) lower bounds for fuchsian groups $\Gamma$ of finite volume with a parabolic element and for $\left\|\boldsymbol{\Theta}_{q}\right\|$ for certain congruence subgroups of the modular group $\Gamma(1)$. We begin with a discussion of the extent to which McMullen's paper is effective.

To begin with, McMullen's proof is by contradiction. Next, the proof is given in detail only for $q=2$, i.e. $A_{2}(\Gamma)$ is the space of quadratic differentials. He states in a brief appendix that Kra's conjecture is similarly provable if $q>2$. This seems reasonable to this writer. For $q=2$, McMullen begins with an element $\psi \in A_{2}(\Gamma)$ of norm one. He then obtains that it is sufficient to show that $\left\|\left.\Theta_{2}\right|_{\Theta_{2}^{-1}(\psi)}\right\|<1$ for any choice of $\psi$. (This is not difficult and was foreshadowed in [P-S, Lemma 1]. But McMullen's strengthening is crucial.) From this point forward, McMullen's proof is effective, that is he shows $\left\|\left.\Theta_{2}\right|_{\Theta_{2}^{-1}(\psi)}\right\| \leq 1-\varepsilon_{\psi}$ where an algorithm for finding $\varepsilon_{\psi}$ really is presented. No example is worked through and the algorithm depends on a choice of a set of neighborhoods (a net) on $H / \Gamma$ that avoids the zeroes of $\psi$. This net gives rise to a Čech graph whose expansion constant is also involved.

Another serious concern is this: using McMullen's technique to compute (or approximate) $\left\|\Theta_{q, \Gamma}\right\|$ amounts to knowing which $\psi$ (or $\psi$ 's) $\in A_{q}(\Gamma) \max -$ imizes $\varepsilon_{\psi}$ (or at least have large $\varepsilon_{\psi}$ 's). McMullen's paper gives no method for locating such "good" $\psi$ 's. Lastly, McMullen's paper does not deal with branched covers. Whether this omission is essential, at least in regard to effectiveness, I cannot say. If it were, that would raise concerns for the modular group and many congruence subgroups.

This paper takes an altogether different approach: for congruence subgroups of $\Gamma(1)$ we display functions $f$ in $A_{q}$ whose Poincare series are "good" $\psi \epsilon$ $A_{q}(\Gamma)$, at least for large $q$. For such $q$ it turns out that both the $A_{q}$ norm of $f$ and the $A_{q}(\Gamma)$ norm of $\Theta_{q, \Gamma}(f)$ have main term $\int_{F}|f| d \mu_{q}$, which approaches $\infty$ with $q$ and error term $O\left(\frac{(2 \pi e)^{q-2}}{q^{q-2}}\right)$ which approaches 0 with $q$, of course. Thus using these functions we can show $\left\|\Theta_{q, \Gamma}\right\| \rightarrow 1$ as $q \rightarrow \infty$, an easy consequence of Theorem 1. That this might be true was an idea of Parson dating back ten years. (It is consistent with her data in [P2].) Last, finding "good" functions in $A_{q}$ is more difficult than finding good $\psi$ 's because the kernel of $\Theta_{q, \Gamma}$ is infinite dimensional. We now define our "good" functions in $A_{q}$. 
For an integer $k \geq 2$ we define two Eisenstein series:

$$
E_{k}(z)=\sum_{A \in \Gamma / \Gamma_{\infty}}\left|A^{\prime} z\right|^{k}
$$

and

$$
G_{k}(z)=\sum_{A \in \Gamma / \Gamma_{\infty}}\left(A^{\prime} z\right)^{k}
$$

Of course, $A^{\prime}(z)=(c z+d)^{-2}$ where $A=\left(\begin{array}{l}\cdot \dot{c} \\ c d\end{array}\right)$, and this gives the more usual definition. $\Delta(z)$ is the unique cusp form of weight 6 on $\Gamma(1)$. (I.e., $\Delta(A z)\left(A^{\prime} z\right)^{6}=\Delta(z)$ is the functional equation. This is usually called weight 12 in number theory.) Of course

$$
\Delta(z)=e(z) \prod_{n=1}^{\infty}(1-e(m z))^{24}=\sum_{n=1}^{\infty} \tau(n) e(n z)
$$

Here $e(z)=e^{2 \pi i z}$ and $\tau(1)=1, \tau(2)=-24, \ldots$ is the Ramanujan $\tau$ function.

Our "good" functions in $A_{q}$ are nontrivial cusp forms of weight $p$ where $p \leq q-2$. (While the choice of $p$ will vary with the application, we will henceforth assume that $p$ is taken large enough so that such cusp forms exist. Thus $q \geq p+2 \geq 8$ in the case of $\Gamma=\Gamma(1)$.) We will denote these $f$ or $f_{p}$. If $\Gamma$ is a congruence subgroup we will specify $f_{p}=\Delta \cdot G_{p-6} \cdot A_{q}$ only requires analyticity and so it is here that McMullen's remark in the second paragraph of the "Guide to the paper" to the effect that a "good" function must "mimic... $\psi$ " motivated our choice. Choosing a cusp form of weight $q$ would be ideal, of course, except that such a function cannot have finite $A_{q^{-}}$ norm. (In Parson's work [P2] she looks for "good" $f \in A_{q}$ that are squares, thus facilitating computation of the $A_{q}$-norm.)

We get our lower bounds for $\left\|\Theta_{q, \Gamma}\right\|$ by getting lower bounds for $\frac{\left\|\boldsymbol{\Theta}_{q, \Gamma}\left(f_{p}\right)\right\|_{A_{q}(\Gamma)}}{\left\|f_{p}\right\|_{A_{q}}}$. The choice of $p$ as the smallest value for which there is a cusp form on $\Gamma$ of weight $p$ leads us to this consequence of Theorem 1: let $\Gamma$ be a Fuchsian group containing a parabolic element (assumed to be $\left.\left(\begin{array}{ll}1 & 1 \\ 0 & 1\end{array}\right)\right)$ and with finite volume. Then $\left\|\Theta_{q, \Gamma}\right\| \geq 1-O\left(\frac{(2 \pi e)^{q-2}}{q^{q-2}}\right)$.

The error term arises as follows: We will show that $\left\|\Theta_{q, \Gamma}\left(f_{p}\right)\right\|_{A_{q}(\Gamma)} \geq$ $\int_{F}\left|f_{p}\right| d \mu_{q}-\varepsilon_{1}-\varepsilon_{2}$ and $\left\|f_{p}\right\|_{A_{q}} \leq \int_{F}\left|f_{p}\right| d \mu_{q}+\varepsilon_{1}+\varepsilon_{2}$. Here all $\varepsilon_{i} \downarrow 0$ as $q \rightarrow$ $\infty$, and $\int_{F}\left|f_{p}\right| d \mu_{q} \rightarrow \infty$ rapidly as $q \rightarrow \infty$. Thus $\left\|\Theta_{q, \Gamma}\right\| \geq 1-\frac{2\left(\varepsilon_{1}+\varepsilon_{2}\right)}{\int_{F}\left|f_{p}\right| d \mu_{q}+\varepsilon_{1}+\varepsilon_{2}}$.

After completing an initial version of Theorem 1, I communicated it to McMullen, among others. He quickly replied with a short write up [M2], elegant but simple, of a geometric argument showing the following theorem. 
Theorem (McMullen). Let $X=H / \Gamma$ be a Riemann surface (unbranched?M.S.) of width $r$. Then $\left\|\Theta_{q, \Gamma}\right\| \geq 1-2\left(1-R^{2}\right)^{q-1}$ where $R=\tanh (r / 2)$. Here the width of Riemann surface is the hyperbolic radius of the largest imbedded "round" disc. Because the width at any cusp of $H / \Gamma(N)$ is essentially $\log N$ (the area of the largest ball in the horocyclic neighborhood of the cusp), he gets

$$
\left\|\Theta_{q, \Gamma(N)}\right\| \geq 1-2 \cdot\left[\frac{4 \sqrt{1+N}}{2+N+2 \sqrt{1+N}}\right]^{q-1} .
$$

(This would apply, mutatis mutandis, to any $\Gamma$ with $\Gamma(N) \subseteq \Gamma \subseteq \Gamma_{0}(N)$; though as indicated above branch points might raise problems.)

This last result enables McMullen to show that $\left\|\Theta_{q, \Gamma}\right\| \rightarrow 1$ as $N \rightarrow \infty$ for fixed $q$ with $\Gamma(N) \subseteq \Gamma \subseteq \Gamma_{0}(N)$. The method of the present paper can also produce such a result, as I found after reading McMullen's result. Our error term has order $N^{-q}$ in place of $N^{\frac{-(q-1)}{2}}$ of McMullen. However, we require $q>35$ for the error term to approach zero:

Theorem 2. Let $\Gamma(N) \subseteq \Gamma \subseteq \Gamma_{0}(N)$. Then $\left\|\Theta_{q, \Gamma}\right\| \geq 1-O\left(N^{35-q}\right)$.

This is proved using $f_{p}=\Delta \cdot G_{p-6}$ with $p=[q / 2]$ and then estimating the norms of Theorem 1.

This idea has a further amusing application. Lemma 2, the beginning of our estimate of $\left\|\Theta_{q, \Gamma}\left(f_{p}\right)\right\|$, obviously implies this norm is greater than 0 . Since a nonzero linear combination of $p$-forms is itself a $p$-form, we get that if $\left\langle f_{p 1}, \ldots, f_{p t}\right\rangle$ span $A_{p}(\Gamma)$ then $\left\langle\Theta_{q, \Gamma}\left(f_{p 1}\right), \ldots, \Theta_{q, \Gamma}\left(f_{p t}\right)\right\rangle$ are linearly independent in $A_{q}(\Gamma)$. If $\operatorname{dim} A_{q}(\Gamma)=\operatorname{dim} A_{p}(\Gamma)$, which can easily happen for the full modular group and $q-11 \leq p \leq q-2$ for example, then this set of Poincaré series spans $A_{q}(\Gamma)$.

We close the introduction with our definition of the maximum cusp width denoted $c_{\infty}$. In results such as Lemma 3 , the cusp width may be thought of as the euclidean radius of the largest (by hyperbolic area) round disk in the horocyclic neighborhood of the cusp after this neighborhood is mapped into $\{\operatorname{Im} z \geq 1\}$. For congruence subgroups, up to a universal computable multiplicative constant, this is the number of $\Gamma(1)$ fundamental regions in the "bouquet" at the cusp. The maximum is taken over all cusps of $\Gamma$. For example for $\Gamma(N), c_{\infty}=N$.

The author would like to thank Jonathan Huntley for pointing out a crucial oversight in the proof of Lemma 7 as the final manuscript was being written. Thanks also to the referee for suggesting many, many improvements and clarifications.

Lemma 1. Let $f$ be a cusp form on $\Gamma$ of weight $p \leq q-2$. Then $f \in A_{q}$ and $\|f\|_{A_{q}}=\int_{F}|f| \cdot E_{q-p} \cdot d \mu_{q}$. 
Proof.

$$
\begin{aligned}
\int_{0}^{1} \int_{0}^{\infty}|f| d \mu_{q} & =\sum_{A \in \Gamma / \Gamma_{\infty}} \int_{A F}|f| y^{q-2} d x d y \\
& =\sum_{A} \int_{F}|f \circ A|(y \circ A)^{q} \frac{d x d y}{y^{2}}=\sum_{A} \int_{F}|f \circ A|\left|A^{q q}\right| y^{q-2} d x d y \\
& =\int_{F}\left(\sum_{A}|f|\left|A^{(q-p)}\right|\right) d \mu_{q}=\int_{F}|f| \cdot E_{q-p} \cdot d \mu_{q}<\infty
\end{aligned}
$$

(This last inequality depends on $f$ being a cusp-form.)

Lemma 2. Let $f$ be as in Lemma 1. Then $\left\|\Theta_{q}(f)\right\|_{A_{q}(\Gamma)}=\int_{F}|f| \cdot\left|G_{q-p}\right| \cdot d \mu_{q}$. Proof.

$$
\begin{aligned}
\int_{F}\left|\sum_{A \in \Gamma / \Gamma_{\infty}}(f \circ A)\left(A^{q q}\right)\right| d \mu_{q} & =\int_{F}\left|\sum_{A} f \cdot A^{(q-p)}\right| d \mu_{q} \\
& =\int_{F}|f|\left|G_{q-p}\right| d \mu_{q} .
\end{aligned}
$$

In what follows, $c_{f}=\max |f| y^{p}$. This is finite when $f$ is a cusp form of weight $p$.

Lemma 3. Let $F=F_{h} \dot{\cup} F_{l}$, where $F_{l}=F \cap\{\operatorname{Im}(z) \leq 1\}$. Let $q>p+2$. Then for any $\delta>0, \int_{F_{l}}|f| \cdot E_{q-p} \cdot d \mu_{q} \leq \frac{c_{f} \cdot c_{\delta, \Gamma, p}}{q^{1-\delta}} \equiv: \varepsilon_{1}$.

Proof. Kubota [K, p. 13] gives the estimate $E_{s} \cdot y^{s}=O\left(y^{s-1}\right)$, where $z=x+$ $i y \in F_{l}$ lies in a horocyclic neighborhood of a noninfinite cusp and the implied constant is independent of $s$. This simple euclidean area argument given there shows that this implied constant is $\leq$ the maximum cusp width of $\Gamma$. Thus $\int_{\text {horo nbds }}\left|f_{p}\right| E_{q-p} d \mu_{q}=\int\left|f_{p}\right| y^{p} E_{q-p} y^{q-p} d \mu_{0} \leq c_{\infty} \cdot c_{f} \cdot \int_{0}^{1} y^{q-p-3} d y=c_{\infty}$. $c_{f} / q-p-2$.

The portion of $F_{l}$ that lies away from all the horocyclic cusp nbds is a compact set $\mathscr{C}$ depending only on $\Gamma$. For some $\delta>0$ let $\mathscr{C}_{1}$ be the set of $z \in \mathscr{C}$ with some $\Gamma$-equivalent of $z$ lying above $\left(1-1 /\left(q^{1-\delta}\right)\right)$ and let $\mathscr{C}_{2}=F_{l}-\mathscr{C}_{1}$. On $\mathscr{C}_{1}$ we have

$$
\int_{\mathscr{E}_{1}}\left|f_{p}\right| E_{q-p} d \mu_{q}=\int_{\mathscr{E}_{1}}\left|f_{p}\right| y^{p} E_{q-p} y^{q-p} d \mu_{0} \leq c_{f} \cdot K_{\Gamma, \delta} \cdot 1 / q^{1-\delta}
$$

because $E_{s} y^{s}$ is bounded for $y \in F_{l}, s \geq 2$; and the (invariant) integral may be taken over a set in $\left\{z \mid 1 \geq y \geq 1-1 /\left(q^{1-\delta}\right)\right\}$. 
For $z \in \mathscr{C}_{2}$ we have $\operatorname{Im}(A z) \leq 1-1 / q^{1-\delta}$, and

$$
\begin{aligned}
E_{q-p} y^{q-p} & =\sum_{A \in \Gamma / \Gamma_{\infty}}(y(A z))^{q-p} \leq\left(1-\frac{1}{q^{1-\delta}}\right)^{q-p-2} \cdot \sum_{A} y^{2}(A z) \\
& =\left(1-\frac{1}{q^{1-\delta}}\right)^{q} \cdot\left(1-\frac{1}{q^{1-\delta}}\right)^{-p-2} \cdot \sum_{A} y^{2}(A z) \leq H_{\Gamma, p} \cdot e^{-q^{\delta}}
\end{aligned}
$$

and so

$$
\begin{aligned}
\int_{\mathscr{E}_{2}}\left|f_{p}\right| E_{q-p} d \mu_{q} & =\int_{\mathscr{E}_{2}}\left|f_{p}\right| y^{p} E_{q-p} \cdot y^{q-p} d \mu_{0} \\
& \leq c_{f} H_{\Gamma, p} e^{-q^{\delta}} \int_{\mathscr{E}_{2}} d \mu_{0}=c_{f} L_{\Gamma, \delta, p} e^{-q^{\delta}} .
\end{aligned}
$$

So we finally get

$$
\int_{F_{l}}\left|f_{p}\right| E_{q-p} d \mu_{q} \leq \frac{c_{\infty} c_{f}}{q-p-2}+\frac{c_{f} K_{\Gamma, \delta}}{q^{1-\delta}}+c_{f} L_{\Gamma, \delta, p} e^{-q^{\delta}} \leq \frac{c_{f} c_{\Gamma, \delta, p}}{q^{1-\delta}} .
$$

Corollary 1. $\int_{F_{l}}\left|f \| G_{q-p}\right| d \mu_{q} \leq \varepsilon_{1}$.

Lemma 4. $\left|\int_{F_{h}}\right| f\left|E_{q-p} d \mu_{q}-\int_{F_{h}}\right| f\left|d \mu_{q}\right| \leq c_{f}\left(\frac{\sqrt{2} e}{(1+\varepsilon)^{2(q-p-1)}(q-p)^{1 / 2}}+\frac{2}{q-p-1}\right) \equiv: \varepsilon_{2}$.

Proof. We use an amplification of the technique suggested in Kubota [K, p. 13]. Let $s \geq 2$. We begin by estimating $E_{s} \cdot y^{s}-y^{s}$ for $z=x+i y \in F_{h}$. For these $y$ the horocycle $\{\operatorname{Im} z \geq y\}$ is mapped by $\{A\} \in \Gamma / \Gamma_{\infty}$ onto a disjoint set of horocycles with radii $r_{A}<\frac{1}{2 y}$ and cusp in $\left(-\frac{1}{2}, \frac{1}{2}\right)$, say. Because of this, the fact that $y \geq 1$, and $\Gamma_{\infty}=\left\langle\left(\begin{array}{ll}1 & 1 \\ 0 & 1\end{array}\right)\right\rangle$, we get $A=\left(\begin{array}{l}\cdots \\ c\end{array}\right)$ has $|c| \geq 1$ and

$$
\#\left\{A\left|A=\left(\begin{array}{l}
. \\
c
\end{array}\right) \in \Gamma / \Gamma_{\infty},\right| c \mid=1\right\} \leq 2 .
$$

(This is like Shimizu's Lemma [Sh].) Let us call $\mathscr{B}$ this set of elements of $\Gamma / \Gamma_{\infty} \cdot$ Clearly

$$
E_{s} \cdot y^{s}-y^{s}=\sum_{\substack{A \notin \mathscr{B} \\ A \notin \text { id }}} y_{A}^{s}+\sum_{A \in \mathscr{B}} y_{A}^{s} \text {. }
$$

Here $y_{A} \equiv: \operatorname{Im}(A z)$. Next note that if $A$ is in the first sum, $A=\left(\begin{array}{l}. \\ c\end{array}\right)$ has $|c|>1+\varepsilon$, where $\varepsilon$ depends only on $\Gamma$. Let $\mathscr{A}$ be this set of $A \in \Gamma / \Gamma_{\infty}$.

For $A \in \mathscr{A}$, we have $2 r_{A} \leq \frac{1}{(1+\varepsilon)^{2} y}$. Let $0<\delta<1$, where we shall choose a specific $\delta$ near one later. Let $T_{A, \delta}$ be the points of the horocycle at $A(\infty)$ that lie above $(1+\delta) r_{A}$. The area of $T_{A, \delta}$ is $r_{A}^{2}\left(\arcsin \sqrt{1-\delta^{2}}-\delta \sqrt{1-\delta^{2}}\right)$. For $\delta$ near one this is $(1-\delta)^{3 / 2} \sqrt{2} r_{A}^{2}+$ higher powers of $(1-\delta)$. Now

$$
\sum_{A \in \mathscr{A}} y_{A}^{s} \leq 4 \sum_{A \in \mathscr{A}} y_{A}^{s-2} r_{A}^{2} \leq\left(\frac{2}{1-\delta}\right)^{3 / 2} \sum_{A \in \mathscr{A}} y_{A}^{s-2} \text { area } T_{A, \delta}
$$


We may assume WLOG that $y_{A} \in\left((1+\delta) r_{A}, 2 r_{A}\right)$, as this makes the sum bigger. We have

$$
(1+\delta)^{s-2} \sum_{A \in \mathscr{A}} r_{A}^{s-2} \text { area } T_{A, \delta} \leq \sum_{A \in \mathscr{A}} y_{A}^{s-2} \text { area } T_{A, \delta} \leq 2^{s-2} \sum_{A \in \mathscr{A}} r_{A}^{s-2} \text { area } T_{A, \delta}
$$

But

$$
\sum_{A \in \mathscr{A}} y_{A}^{s-2} \text { area } T_{A, \delta} \leq \sum_{A \in \mathscr{A}} \int_{T_{A, \delta}} t^{s-2} d x d t \leq \frac{y^{1-s}}{(s-1)(1+\varepsilon)^{2(s-1)}},
$$

recalling that $2 r_{A} \leq \frac{1}{(1+\varepsilon)^{2} y}$. Thus

$$
\begin{aligned}
& 2^{s-2} \sum_{\mathscr{A} \in A} r_{A}^{s-2} \text { area } T_{A, \delta} \\
& \quad \leq\left(\frac{2}{1+\delta}\right)^{s-2} \sum_{A \in \mathscr{A}} y_{A}^{s-2} \text { area } T_{A, \delta} \leq\left(\frac{2}{1+\delta}\right)^{s-2} \frac{y^{1-s}}{(s-1)(1+\varepsilon)^{2(s-1)}} .
\end{aligned}
$$

Finally,

$$
\sum_{A \in \mathscr{A}} y_{A}^{s} \leq\left(\frac{2}{1-\delta}\right)^{3 / 2} \cdot\left(\frac{2}{1+\delta}\right)^{s-2} \cdot \frac{y^{1-s}}{(1+\varepsilon)^{2(s-1)}(s-1)} .
$$

Now let $\delta=1-\frac{1}{s}$. We have

$$
\begin{aligned}
\sum_{A \in \mathscr{A}} y_{A}^{s} & \leq \sqrt{2} e \cdot \frac{s^{1 / 2}}{(1+\varepsilon)^{2(s-1)}} \cdot y^{1-s} \cdot\left|\int_{F_{h}}\right| f\left|E_{q-p} d \mu_{q}-\int_{F_{h}}\right| f\left|d \mu_{q}\right| \\
& \leq \int_{F_{h}}|f| y^{p}\left|E_{q-p} y^{q-p}-y^{q-p}\right| \frac{d x d y}{y^{2}} \\
& \leq c_{f} \cdot \int_{F_{h}}\left(\sum_{A \in \mathscr{A}} y_{A}^{q-p}+\sum_{A \in \mathscr{B}} y_{A}^{q-p}\right) \frac{d x d y}{y^{2}} \\
& \leq \frac{\sqrt{2} e c_{f}}{(1+\varepsilon)^{2(q-p-1)}(q-p)^{1 / 2}}+c_{f} \int_{F_{h}} \sum_{A \in \mathscr{B}} y_{A}^{q-p} \frac{d x d y}{y^{2}}
\end{aligned}
$$

This last integral is bounded by $\frac{2 c_{f}}{q-p-1}$, since $y_{A} \leq \frac{1}{y}$ for $A \in \mathscr{B}$, a fact that follows from $|c|=1$, and that $|\mathscr{B}| \leq 2$. In summary we have shown:

$$
\left|\int_{F_{h}}\right| f\left|E_{q-p} d \mu_{q}-\int_{F_{h}}\right| f\left|d \mu_{q}\right| \leq c_{f}\left(\frac{\sqrt{2} e}{(1+\varepsilon)^{2(q-p-1)}(q-p)^{1 / 2}}+\frac{2}{q-p-1}\right) .
$$

Lemma 5. With $f$ as in Lemma 1, assume further that $f(z)=f(z+1)$ and that $f$ has Fourier expansion $\sum_{n=1}^{\infty} a_{n} e(n z) ; a_{1}=1$. If

$$
q \geq \max (2+2 \pi, p+2),
$$

then $\int_{F_{h}}|f| y^{q-2} d x d y \geq\left(\frac{q-2}{2 \pi e}\right)^{q-2}\left[1-c_{f} c_{p} e^{-2 q} /(q-2)\right]$ where $c_{p}=: 2^{p} p ! p e^{4}$. 
Proof.

$$
|f(z)|=e^{-2 \pi y}\left|1+a_{2} e(z)+a_{3} e(2 z)+\cdots\right| \geq e^{-2 \pi y}\left|1-c_{f} \sum_{n=2}^{\infty} n^{p} e^{-2 \pi(n-1) y}\right|
$$

where we have used the "trivial" (Hecke) estimate $\left|a_{n}\right| \leq c_{f} n^{p}$. We have

$$
\sum_{n=2}^{\infty} n^{p} e^{-n(q-2)} \sim \int_{2}^{\infty} x^{p} e^{-x(q-2)} d x \leq 2^{p} p ! p e^{4} e^{-2 q} /(q-2)=c_{p} e^{-2 q} /(q-2)
$$

using formula $3.351(2)$ in [G-R]. So for $y \in\left[\frac{q-2}{2 \pi}, \frac{q-2}{2 \pi}+1\right]$ we get

$$
|f(x+i y)| \geq e^{-(q-2+2 \pi)}\left[1-c_{f} c_{p} e^{-2 q} /(q-2)\right]
$$

and

$$
\begin{gathered}
\int_{F_{h}}|f| y^{q-2} d x d y \geq\left(\int_{\frac{q-2}{2 \pi}}^{\frac{q-2}{2 \pi}+1} y^{q-2} d y\right) e^{-(q-2+2 \pi)}\left[1-c_{f} c_{p} e^{-2 q} /(q-2)\right] \\
\geq\left(\frac{q-2}{2 \pi e}\right)^{q-2} \cdot e^{-2 \pi} \cdot\left[1-c_{f} c_{p} e^{-2 q} /(q-2)\right] .
\end{gathered}
$$

This becomes nontrivial for $q$ so large that the last factor on the RHS is positive. Call this value $Q_{0}=Q_{0}(\Gamma)$.

Lemma 6. $\left|\int_{F_{h}}\right| f|| G_{q-p}\left|d \mu_{q}-\int_{F_{h}}\right| f\left|d \mu_{q}\right| \leq \varepsilon_{2}$.

Proof.

$$
y^{s}-\sum_{\substack{A \neq \text { id } \\ A \in \Gamma / \Gamma_{\infty}}} y_{A}^{s} \leq\left|G_{s}\right| y^{s} \leq y^{s}+\sum_{\substack{A \neq \text { id } \\ A \in \Gamma / \Gamma_{\infty}}} y_{A}^{s} .
$$

The lemma now follows using the $\mathscr{A}, B$ decomposition just as did Lemma 4.

At this point we can prove

Theorem 1. Let $\Gamma$ be a Fuchsian group containing a parabolic element and with finite volume. Suppose WLOG that $\infty$ is a cusp with stabilizer generated by $z \rightarrow z+1$. Let $p$ be the smallest weight with a nontrivial cusp form $f_{p}$ on $\Gamma$. Assume-only for convenience-that $a_{f_{p}}(1)=1$. Then for

$$
q \geq \max \left(2+2 \pi, p+2, Q_{0}\right)
$$

we have $\left\|\theta_{q, \Gamma}\right\|>1-O\left(\frac{\left(\varepsilon_{1}+\varepsilon_{2}\right)(2 \pi e)^{q-2}}{(q-2)^{q-2}}\right)$.

Proof. For $q \geq p+2$ we have $f_{p} \in A_{q}$ (Lemma 1) and

$$
\left\|f_{p}\right\|_{A_{q}}=\int_{F_{h}}\left|f_{p}\right| E_{q-p} d \mu_{q}+\int_{F_{l}}\left|f_{p}\right| E_{q-p} d \mu_{q} \leq \int_{F_{h}}\left|f_{p}\right| d \mu_{q}+\varepsilon_{1}+\varepsilon_{2}
$$


(Lemmas 1, 3 and 4). Next,

$$
\begin{aligned}
\left\|\Theta_{q}\left(f_{p}\right)\right\|= & \int_{F}\left|f_{p}\right|\left|G_{q-p}\right| d \mu_{q} \geq \int_{F_{h}}\left|f_{p}\right|\left|G_{q-p}\right| d \mu_{q} \\
& -\int_{F_{l}}\left|f_{p}\right|\left|G_{q-p}\right| d \mu_{q} \geq \int_{F_{h}}\left|f_{p}\right| d \mu_{q}-\varepsilon_{1}-\varepsilon_{2}
\end{aligned}
$$

by Corollary 1 and Lemma 6 . Therefore

$$
\left\|\Theta_{q, \Gamma}\right\| \geq \frac{\left\|\Theta_{q}\left(f_{p}\right)\right\|}{\left\|f_{p}\right\|} \geq \frac{\int_{F_{h}}\left|f_{p}\right| d \mu_{q}-\varepsilon_{1}-\varepsilon_{2}}{\int_{F_{h}}\left|f_{p}\right| d \mu_{q}+\varepsilon_{1}+\varepsilon_{2}} \geq 1-\frac{2\left(\varepsilon_{1}+\varepsilon_{2}\right)}{\int_{F_{h}}\left|f_{p}\right| d \mu_{q}+\varepsilon_{1}+\varepsilon_{2}} .
$$

Thus by Lemma 5 ,

$$
\left\|\Theta_{q, \Gamma}\right\| \geq 1-O\left(\frac{\left(\varepsilon_{1}+\varepsilon_{2}\right)(2 \pi e)^{q-2}}{(q-2)^{q-2}}\right) .
$$

We now specialize to groups $\Gamma$ that are between $\Gamma_{0}(N)$ and $\Gamma(N)$. What is crucial for us about these groups is (a) $c_{\infty} \leq N$ (b) $A=\left(\begin{array}{c}c \\ c\end{array}\right) \Rightarrow N \mid c$. These two properties give us a version of Theorem 1 valid for fixed $q$ and $N \rightarrow \infty$.

The following lemma assumes that $q$ is even. This is a purely technical assumption. If $q$ is odd, select $f$ of weight $q-\left[\frac{q}{2}\right]$, replace $2 k$ by $q$, and $E_{k}$ by $E_{\left[\frac{q}{2}\right]}$.

Lemma 7. Let $\Gamma(N) \subseteq \Gamma \subseteq \Gamma_{0}(N)$ and $[\Gamma: \Gamma(N)]=M$. Let $f$ be a cusp form of weight $k=\left[\frac{q}{2}\right]$. Then $\int_{F}|f| d \mu_{2 k}-\varepsilon_{3} \leq\|f\|_{A_{2 k}} \leq \int_{F}|f| d \mu_{2 k}+\varepsilon_{3}$ where $\varepsilon_{3} \equiv: d_{f} \cdot N^{1-2 k} \cdot K_{1} \cdot M$ and $d_{f}=\int_{F}|f| d \mu_{0}$.

Proof. According to Lemma 1, which extends mutatis mutandis to $\Gamma_{\infty}=\left\langle\left(\begin{array}{ll}1 & N \\ 0 & 1\end{array}\right)\right\rangle$, we must estimate $\int_{F}|f| E_{k} d \mu_{2 k}$. We begin by noting that WLOG $c=c_{1} N$ with $c_{1}>0, d=d_{1} N+m$ with $0 \leq m \leq N-1$. Then

$$
E_{k}=1+\sum_{\substack{A \neq \text { id } \\ A \in \Gamma / \Gamma_{\infty}}} \frac{1}{|c z+d|^{2 k}}=1+N^{1-2 k} \sum_{m} \sum_{c_{1}>0}^{\infty} \sum_{d_{1}=-\infty}^{\infty}\left|c_{1} z+\frac{m}{N}+d_{1}\right|^{-2 k}
$$

with the notation of Lemma 1 . We need to extract a bit more from the lattice estimate for Eisenstein series [A, p. 7] given in Apostol. This we do by noting that $c_{1} \neq 0$ removes an entire line of lattice points from the double sum and thus $(z, 0)$ is always the lattice point closest to $(0,0)$ in the $\left(c_{1}, d_{1}\right)$ lattice. Thus the triple sum is always less than $M \cdot K_{1} \cdot y^{-2 k}$. This gives

$$
y^{2 k} E_{k} \leq y^{2 k}+N^{1-2 k} \cdot K_{1} \cdot M .
$$

If we write $d_{f}=\int_{F}|f| \frac{d x d y}{y^{2}}$, which is finite as $f$ is a cusp form, we have

$$
\int_{F}|f| E_{k} d \mu_{2 k} \leq \int_{F}|f| d \mu_{2 k}+d_{f} \cdot N^{1-2 k} \cdot M \cdot K_{1} .
$$

The lower bound proof is similar. 
Corollary 2. With $f$ and $\Gamma$ as above

$$
\int_{F}|f| d \mu_{2 k}-\varepsilon_{3} \leq \int_{F}|f|\left|G_{k}\right| d \mu_{2 k} \leq \int_{F}|f| d \mu_{2 k}+\varepsilon_{3}
$$

where $\varepsilon_{3}$ is as in Lemma 7.

Theorem 2. Let $\Gamma(N) \subseteq \Gamma \subseteq \Gamma_{0}(N)$. Then $\left\|\Theta_{q, \Gamma}\right\| \geq 1-O\left(N^{35-q}\right)$; where the implied constant is independent of $\Gamma$ but not $q$.

Proof. Choose $f$ as in Lemma 7. Then

$$
\frac{\left\|\boldsymbol{\Theta}_{q}(f)\right\|}{\|f\|} \geq \frac{\int_{F}|f| d \mu_{2 k}-\varepsilon_{3}}{\int_{F}|f| d \mu_{2 k}+\varepsilon_{3}} \geq 1-\frac{2 \varepsilon_{3}}{\int_{F}|f| d \mu_{2 k}+\varepsilon_{3}} .
$$

We now specialize to $f=\Delta \cdot G_{k-6}$, with $\Delta(z)=e(z) \prod_{m=1}^{\infty}(1-e(m z))^{24}$. We must estimate $d_{f}=\int_{F}|f| d \mu_{0}$ and $\int_{F}|f| d \mu_{2 k}$ for this choice of $f$.

Write $F=F_{h} \dot{\cup} F_{l}$ as in Lemma 3. (Care must be taken as now $F$ depends on $N$.)

$$
\begin{aligned}
\int_{F_{h}}|f| d \mu_{0} & \leq \int_{F_{h}}|\Delta| E_{k-6} d \mu_{0} \leq c_{1} \int_{F_{h}}|\Delta| y^{6-k} d \mu_{0} \\
& =c_{1} N \int_{1}^{\infty} \int_{0}^{1}|\Delta| y^{4-k} d x d y=c_{2} N
\end{aligned}
$$

Restating,

$$
\int_{F_{h}}|f| d \mu_{0}<c_{2} N
$$

Consider the horocyclic nbd $C$ of $\infty$ given by $y \geq \frac{\sqrt{3}}{2}$. This just touches the lowest points-elliptic fixed points of order three-of $\Gamma(1)$. Since this horocycle contains the bouquet at $\infty$, the $\Gamma$-translates of $C$ cover $F$. Further, each point of $F$ lies in at most two translates of $C$. The euclidean argument referred to at the beginning of Lemma 3 now shows $E_{s} \cdot y^{s}=O\left(y^{s-1}\right)$, where the implied constant is at most $4 / \sqrt{3}$ times bigger. ( 2 is for the double coverage and $2 / \sqrt{3}$ is for the wider horocycles.) I.e., for this $\Gamma$ we have $E_{s} \cdot y^{s} \leq \frac{4}{\sqrt{3}} N y^{s-1}$ on all of $F_{l}$ so that

$$
\int_{F_{l}}|f| d \mu_{0} \leq \int_{F_{l}}|\Delta| E_{k-6} d \mu_{0} \leq c_{3} N \int_{F_{l}}|\Delta| \frac{d x d y}{y^{3}} .
$$
and

Let $T_{a / c}$ be a $\Gamma(1)$ FR intersecting $F_{l}$. Here $a / c$ is the real cusp of $T_{a / c}$

$$
A=\left(\begin{array}{ll}
a & \cdot \\
c & d
\end{array}\right) \in \Gamma(1): \infty \rightarrow \frac{a}{c}
$$


Then, with $S$ being the $\Gamma(1)$ SFR,

$$
\begin{aligned}
\int_{T_{a / c}} & |\Delta| \frac{d x d y}{y^{3}}=\int_{S}|\Delta(A z)| y^{-1}(A z) d \mu_{0}=\int_{S}|\Delta(z)|\left|A^{\prime} z\right|^{-7} y^{-1} d \mu_{0} \\
& =\int_{S}|\Delta(z)||c z+d|^{14} y^{-1} d \mu_{0}=|c|^{14} \int_{S}|\Delta(z)|\left|\frac{z+d / c}{y}\right|^{14} y^{11} d x d y \\
& \leq N^{28} \int_{S}|\Delta| y^{11} d x d y=c_{4} N^{28} .
\end{aligned}
$$

(Here we have used that facts that we may assume $|c|<N$ and $|d / c|<N / 2$. This may split up $F_{l}$.) As there are at most $N^{3}$ fundamental regions of $\Gamma(1)$ intersected, we have

$$
\int_{F_{l}}|f| d \mu_{0} \leq c_{5} N^{32}
$$

and combining (2) and (3) we get

$$
\int_{F}|f| d \mu_{0} \leq c_{6} N^{32}
$$

Thus $\varepsilon_{3}$ of Lemma 7 satisfies

$$
\varepsilon_{3} \leq_{k} N^{36-2 k} \text {. }
$$

It remains to obtain a lower bound for $\int_{F}|f| d \mu_{2 k}$. We use the trivial estimate $\int_{F}|f| d \mu_{2 k} \geq \int_{F_{h}}|f| d \mu_{2 k} \geq c_{7} N$, which gives $\left\|\Theta_{q, \Gamma}\right\| \geq 1-O\left(N^{35-q}\right)$, when combined with (4).

\section{REFERENCES}

[A] Tom M. Apostol, Modular functions and Dirichlet series in number theory, Springer-Verlag, New York, 1976.

[G-R] I. S. Gradshteyn and I. M. Ryzhik, Table of integrals, series, and products, Academic Press, New York, 1965.

[K] Tomio Kubota, Elementary theory of Eisenstein series, Halsted Press, New York, 1973.

[M1] Curt McMullen, Amenability, Poincaré series and quasi-conformal maps, Invent. Math. 97 (1989), 95-127.

[M2] L Letter to author (dated August 24, 1988).

[P-S] L. Alayne Parson and Mark Sheingorn, Bounding the norm of the Poincare $\Theta$-operator, Analytic Number Theory, M. I. Knopp, ed., Lecture Notes in Math., vol. 899, SpringerVerlag, Berlin, 1981, pp. 422-441.

[P1] L. Alayne Parson, A lower bound for the norm of the Poincare O-operator, Math. Comp. 41 (1983), 683-685.

[P2] On the Poincaré O-operator, Complex Variables: Theory and Applications 6 (1986), 135-148.

[Sh] H. Shimizu, On discontinuous groups operating on the product of the upper half planes, Ann. of Math. (2) 77 (1963), 33-71.

[St] Kurt Strebel, On the lifts of extremal quasiconformal mappings, J. Analyse Math. 31 (1977), 191-203.

Department of Mathematics, Baruch College (CUNY), New York, New York 10010 E-mail address: mshbb@cunyvm.cuny.edu 